\title{
Palliativmedizin soll extrabudgetär vergütet werden - und es gibt neue Leistungen
}

_ Ab dem 1. Oktober 2017 werden neue Abrechnungspositionen für Leistungen bei Palliativpatienten in den EBM eingeführt. Konkret werden das die Nr. 37300 für die palliativmedizinische Ersterhebung, die Nr. 37302 für eine Koordinationspauschale, die Nr. 37317 für die telefonische Erreichbarkeit und Besuchsbereitschaft sowie die Nr. 37318 für eine telefonische Beratung von mindestens fünf Minuten Dauer sein. Die Abrechnung ist allerdings nur möglich, wenn der Arzt eine sehr zeitaufwändige Weiterbildung durchlaufen hat (Tab. 1).

\section{MMW-KOMMENTAR}

Hausärzte können Leistungen bei Palliativpatienten auch weiterhin ohne Zusatzqualifikation erbringen. Die palliativmedizinische Ersterhebung kann auch nach Nr. 03370 berechnet werden, wobei das Honorar von 5,37 Euro allerdings geringer ist als jenes für die neue Nr. 37 300. Auch die Besuchszuschläge nach den Nrn. 03372 und 03373 sowie die Nr. 03371 für die Betreuung des Patienten in der Praxis stehen weiterhin zur Verfügung. Nicht vergessen sollte man zudem die Nrn.01425 (26,40 Euro) und 01426 (15,86 Euro) für die Erst- bzw. die Folgeverordnung einer spezialisierten Palliativversorgung (SAPV).

Die Nr. 37320 für eine Fallkonferenz z. B. mit Pflegekräften können Hausärzte nun bis zu fünfmal im Krankheitsfall ansetzen - und das ohne Zusatzqualifikation. Die Leistung hat keine Zeitvorgabe.

Die neuen Leistungen werden extrabudgetär vergütet, und auch für die alten Leistungen soll dies ab dem 1. Oktober gelten.

Alle genannten Palliativleistungen können nicht berechnet werden, wenn eine SAPV stattfindet. Neu ist, dass sich der Vertragsarzt erkundigen muss, ob dies der Fall ist. Er trägt somit das finanzielle Risiko einer eventuellen Doppelbehandlung.
Tab. 1 Qualifikationsvoraussetzungen für Hausärzte, die die neuen Palliativleistungen abrechnen wollen

- 2-wöchige Hospitation in palliativmedizinischer Einrichtung oder

- Nachweis über die Betreuung von 15 Palliativpatienten in den vergangenen 3 Jahren

- 40 Kursstunden Palliativmedizin oder

- 20 Kursstunden Schmerztherapie (wenn man bereits 60 Kursstunden Geriatrie und 80 Kursstunden Psychosomatik absolviert hat) oder

- 18 Kursstunden zu definierten Themen (wenn man bereits 80 Kursstunden Schmerztherapie absolviert hat)

- 8 CME-Punkte pro Jahr durch Fortbildungskurse in Palliativmedizin

- Nachweis der Kooperation mit palliativmedizinischen Leistungserbringern, z. B. Pflegedienst

\section{Hier steht eine Anzeige.}

